\title{
BESOIN EN PROTÉINES DU CANETON DE BARBARIE ENTRE LES ÂGES DE 4 ET 11 SEMAINES
}

\author{
13. LECLERCQ et H. de CARVILLE \\ Station de Recherches avicoles, \\ Centre de Recherches de Tours, I. N.R. A., \\ B. P. 1, Nousilly, 37380 Monnaie
}

RÉSUMÉ

Des canetons femelles de Barbaric sont élevés jusclu'ì l'âge de + semaines avec un même aliment. On leur distribue ensuite des aliments ne différant que par leur tanx de matières azotécs totales (taux éncrgétique $12,5 \mathrm{MJ} / \mathrm{kg}$ ). Au cours d'un premier essai on teste 5 taux de MAT : I 8,9, $17,4,16, I_{4}, 6$ et 13,2 p. Ioo: les animaux sont sacrifiés aux âges de ro et 1 I semaines. Dans un deuxième essai les taux expérimentés sont: $16,4,15,5,14,6,13,7$ et I 2,8 p. 100; les abattages ont lieu à 9 et ro semaines.

De ces deux essais on conclut que le besoin protéicue du Caneton est de l'ordre de 15 p. Ioo entre les âges de 4 et 6 semaines. Il s'abaisse à mesure que l'animal vieillit et devient inférieur à $r_{3}$ p. Ioo après l'âge de 8 semaines.

Quand la vitesse de croissance maximum est atteinte, l'indice de consommation n'est pas influencé par le taux protidique, contrairement à ce qui est observé chez lo Poulet.

La dissection de la carcasse révèle que le poids des cuisses atteint dès l'âge de 9 semaines sa valeur maximum. Au contraire le poids cles filets s'accroît avec l'âge en valeur absolue comme en valeur relativc.

\section{IN'TRODUCTION}

I,es besoins alimentaires du canard de Barbarie (Cairina moschata) ne sont guère connus. Il est difficile de s'appuyer sur les résultats acquis chez le canard Pékin (Anas platyrhynchos). Il s'agit en effet d'espèces différentes dont les courbes de croissance présentent des caractéristiques propres. De plus il existe un dimorphisme sexuel très accusé chez le canard de Barbarie absent chez le canard Pékin. Finfin les deux espèces ont des caractéristiques anatomiques différentes. C'est ainsi que le canard de Barbarie possède des masses musculaires (filets et cuisses) plus développées et des dépôts gras moins abondants que le canard Pékin (DELPECH et Mirovanovic, I973).

Nous avons abordé l'étude de l'alimentation du canard de Barbarie par la détermination du besoin en protéines, dont la couverture entraîne les dépenses les plus 
importantes. De plus nous nous sommes intéressés à la période de vie de l'animal s'étendant de l'âge de 4 semaines à celui de l'abattage qui intervient vers la Io ${ }^{\circ}$ semaine. Le caneton consomme en effet durant cette fraction de sa vie $75 \mathrm{p}$. Ioo de l'aliment nécessaire à sa croissance complète.

\section{MATÉRIEI, ET MÉTHODES}

Nous avons procédé à deux expériences portant chacune sur Ioo canctons femelles issus d'un croisement commercial ("Canedins" R 32 G.A.F.C. Grimaud Frères). Les animaux sont élevés par groupes de to sujets en cages grillagées de $1,6 \mathrm{~m} \times 1 \mathrm{~m}$; le fond de la cage étant constitué d'un grillage plastifié à mailles carrées de $2 \mathrm{~cm}$ de côté. Le chauffage est assuré par des réflecteurs à infra-rouges. L'éclairage est permanent pendant 96 heures puis limité à I5 heures par jour. Les tubes fluorescents utilisés sont teintés en rouge.

Tous les canetons sont nourris jusqu'à l'âge de 4 semaines avec un aliment de démarrage dont la formule est rapportée dans le tableau r. Cet aliment est présenté sous forme de granulés d'un diamètre de $2,5 \mathrm{~mm}$. Au-delà de cet âge on constitue 5 lots de 2 cages (répétitions) différant par la teneur en matières azotées totales (MAT) de l'aliment. Celui-ci est distribué sous forme de granulés de $4 \mathrm{~mm}$ de diamètre. Les deux expériences diffèrent par les gammes de teneurs en MAT des aliments distribués au-delà de l'âge de 4 semaines et par l'âge auquel les animaux sont sacri fiés.

TABIEAU I

Composition des régimes expérimentaux ( $\mathrm{c}$ er essai)

(en p. IOO)

\begin{tabular}{|c|c|c|c|c|}
\hline & Démarrage & liégime 19 & Régime 16 & Régime 13 \\
\hline$\ldots \ldots \ldots \ldots$ & 59,0 & 67,0 & 76,0 & 85,0 \\
\hline Tourteau de soja 14 p. 100 & & & & \\
\hline de MAT ............ & 35,0 & 27,0 & 18,5 & 10,0 \\
\hline Huile d'arachide $\ldots \ldots \ldots$ & 1,0 & 1,0 & 0,5 & - \\
\hline Sel .............. & 0,3 & 0,3 & 0,3 & 0,3 \\
\hline Calcimarine .......... & 1,0 & 1,0 & 1,0 & 1,0 \\
\hline Phosphate bicalcique .... & 2,0 & 2,0 & 2,0 & 2,0 \\
\hline DL-méthionine $\ldots \ldots \ldots$ & $0,1 / 5$ & 0,132 & 0,056 & - \\
\hline Oligo-minéraux . . . . . . . & 0,1 & 0,1 & 0,1 & 0,1 \\
\hline Mélange vitaminique .... & 1,6 & 1,6 & 1,6 & 1,6 \\
\hline Taux ćnergétique & & & & \\
\hline $\mathrm{MJ} / \mathrm{kg} \ldots \ldots$ & 11,95 & 12,40 & 12,65 & 12,90 \\
\hline $\mathrm{Kcal} / \mathrm{kg}$ & 2865 & 2960 & 3020 & 3085 \\
\hline $\begin{array}{l}\text { Teneur en MAT mesuréc } \\
\quad(\text { en p. } 100) \ldots \ldots \ldots \ldots\end{array}$ & 21,9 & 18,9 & 16,0 & 13,2 \\
\hline
\end{tabular}

Composition du mélange oligo-minéral (p. $100 \mathrm{~kg}$ ) : oxyde de manganèse $=7 \mathrm{~g}$; oxyde de zinc $=5 \mathrm{~g}$; sulfate ferreux $=0,55 \mathrm{~g}$; carbonate de cuivre $=0,03 \mathrm{~g}$; iodure de potassium $=0,1 \mathrm{~g}$; carbonate de cobalt $=0,03 \mathrm{~g}$; carbonate de calcium $=80,5 \mathrm{~g}$.

Composition du mélange vitaminique (p. $100 \mathrm{~kg}$ ) : vitamine $\mathrm{A}=800000$ $\mathrm{UI}$; vitamine $\mathrm{D}_{3}=100000 \mathrm{UI}$; vitamine $\mathrm{K}=0,2 \mathrm{~g}$; riboflavine $=2 \mathrm{~g}$; acide nicotinique $=5 \mathrm{~g} ;$ acide folique $=0,6 \mathrm{~g} ;$ pyridoxine $=0,6 \mathrm{~g} ;$ biotine $=0,1 \mathrm{~g}$; cyanocobalamine $=0,5 \mathrm{mg}$; pantothénate de calcium $\ldots 2 \mathrm{~g}$; choline $=150 \mathrm{~g}$. 


$$
\text { Expérience } n^{0} 1 \text { (22 mars - } 6 \text { juin) }
$$

On distribue 5 régimes dénommés $19,17,5,16,14,5$ et 13 , selon leur teneur en MAT.

On procède à la fabrication des régimes 19 , 16 et I 3 dont les formules font l'objet du tableau $\mathbf{I}$. Les deux autres régimes sont obtenus par mélange à parties égales des régimes I 9 et 16 d'une part, et ${ }_{5} 5$ et I $_{3}$ d'autre part.

La détermination des teneurs réelles en MAT après analyse par la méthode de Kjeldahl est la suivante :

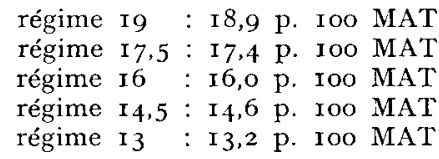

Expérience $n^{\circ} 2$ (I I juillet - I9 septembre)

Les régimes expérimentaux sont dénommés I6, I 5, I 4, I 3 et I2. Ils sont réalisés par mélange en proportions adéquates des deux régimes extrêmes (16 et I2) qui constituent les régimes de base; leur composition exacte est rapportée dans le tableau 2. L'analyse ayant montré que la teneur en MAT du régime 16 était nettement supérieure à celle escomptée, on a ajouté au régime un mélange d'amidon, de cellulose, de minéraux et de vitamines afin d'obtenir la valeur désirée.

\section{TABLEAU 2}

Composition des régimes expérimentaux lors du $2^{\mathrm{e}}$ essai

(en p, IOO)

\begin{tabular}{|c|c|c|}
\hline & Régime 16 & Régine 12 \\
\hline$\ldots \ldots \ldots \ldots \ldots \ldots$ & 57,0 & 86,0 \\
\hline Tourteau de soja (4't p. $1(10$ MAT) & 23,0 & 9,0 \\
\hline Huile d'arachide $\ldots \ldots \ldots \ldots \ldots \ldots$ & $0, t_{t}$ & - \\
\hline Amidon de maïs . . . . . . . . . . & 12,5 & - \\
\hline Cellulose . . . . . . . . . . . . & 2,0 & 一 \\
\hline DL-méthionine $\ldots \ldots \ldots \ldots \ldots \ldots$ & 0,110 & - \\
\hline Sel $\ldots \ldots \ldots \ldots \ldots \ldots \ldots \ldots$ & 0,3 & 0,3 \\
\hline Calcimarine $\ldots \ldots \ldots \ldots \ldots \ldots$ & 1,0 & 1,0 \\
\hline Phosphate bicalcique ............ & 2,0 & 2,0 \\
\hline Oligo-minéraux $(1) \ldots \ldots \ldots \ldots \ldots$ & 0,1 & 0,1 \\
\hline Mélange vitaminique $\left({ }^{1}\right) \ldots \ldots$ & 1,6 & 1,6 \\
\hline \multicolumn{3}{|l|}{ Taux énergétique calculé } \\
\hline $\mathrm{MJ} / \mathrm{kg} \ldots \ldots \ldots \ldots$ & 12,45 & 13,0 \\
\hline $\mathrm{Kcal} / \mathrm{kg} \ldots \ldots \ldots$ & 2970 & 3100 \\
\hline Teneur en MAT mesurće (p. 100) & 16,4 & 12,8 \\
\hline
\end{tabular}

(1) Voir tableau 1.

Compte tenu des valeurs réelles obtenues par dosage, la gamme des teneurs en MAT est la suivante:

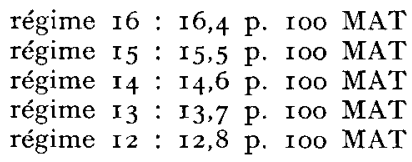



lysine.

Pour tous les régimes on maintient constant et égal à o,80o le rapport acides aminés soufrés/

Dans chaque expérience les animaux sont identifiés par une bague métallique fixée à la membrane alaire. Les pesées individuelles ont lieu aux âges de 4, 6, 8, Io et I I semaines dans le premier essai, 4, 6, 8, 9 et Io au cours du second. On enregistre aux mêmes stades la consommation de l'ensemble de ro anjmaux de chaque cage. A l'âge de 4 semaines les canetons sont systématiquement débecqués; on procède à la section de l'extrémité de la mandibule supérieure en utilisant une pince coupante et en cautérisant au fer rouge.

Des dissections sont effectuées aux âges de io et I I semaines lors de la première expérience, 9 et Io semaines lors de la seconde. A chaque stade la moitié des animaux de chacune des cages est sacrifiée. Le Canard est saigné et plumé. On enlève l'intestin et le pancréas selon la technique classique pour obtenir la carcasse effilée. En divisant le poids de cette carcasse par le poids vif nous calculons le rendement à l'abattage. La cuisse droite est prélevée après dissociation de l'articulation coxo-fémorale et de l'articulation tibio-tarsienne. Les muscles sont sectionnés aux ras du corps. Le filet droit est prélevé en suivant le bréchet, le scapulum, la dernière côte et l'articulation scapulo-humérale.

\section{RÉSULTATS}

\section{I. - Expérience no 1}

Les performances de croissance obtentes lors du premier essai font l'objet du tableau 3. Seul le régime $\mathrm{I}_{3}$ (13,2 $\mathrm{p}$. Ioo MAT) s'avère un peu insuffisant pour assurer la croissance maximale du caneton. Mais la réduction du gain de poids est transitoire et significative seulement entre les âges de 4 et 6 semaines; elle disparaît ensuite. En effet le caneton de plus de 6 semaines réalise sa croissance maximum mesurée par le gain de poids vif avec n'importe lequel des régimes expérimentaux. Bien plus, entre 8 et Io semaines les lots retardés par la déficience protéique entre 4 et 6 semaines présentent une croissance compensatrice.

Dans le tableau 4 nous rassemblons les résultats concernant la consommation d'aliment. Il n'existe guère de différences entre les lots I9, I7, 5, I6 et I4,5. Seul le régime le plus pauvre (I3,2 p. Ioo MAT) provoque une sous-consommation de 4 à 6 et de 6 à 8 semaines ; celle-ci est accompagnée d'un mauvais indice de consommation. Par la suite l'indice de consommation de ce lot devient meilleur que ceux des autres lots du fait de la croissance compensatrice. Il n'existe pas de différence d'indice de consommation entre les différents lots sur l'ensemble de la durée de l'expérience (o à I I semaines).

Le tableau 5 indique le poids des carcasses et fournit les résultats de dissection. Quel que soit le lot et 1'âge, le rendement d'abattage est le même; il atteint toujours une valeur proche de $80 \mathrm{p}$. Ioo. De même le poids de la cuisse atteint dès l'âge de Io semaines une valeur semblable pour tous les lots et qui n'augmente que légèrement au cours de la semaine suivante. Au contraire, le développement des filets ne peut atteindre sa valeur maximum à Io semaines avec le régime le plus pauvre en protéines. Ce retard est compensé entre les âges de Io et I I semaines. Il faut d'ailleurs remarquer que contrairement à la cuisse, le filet tend à augmenter de poids dans tous les lots entre Io et I I semaines. 

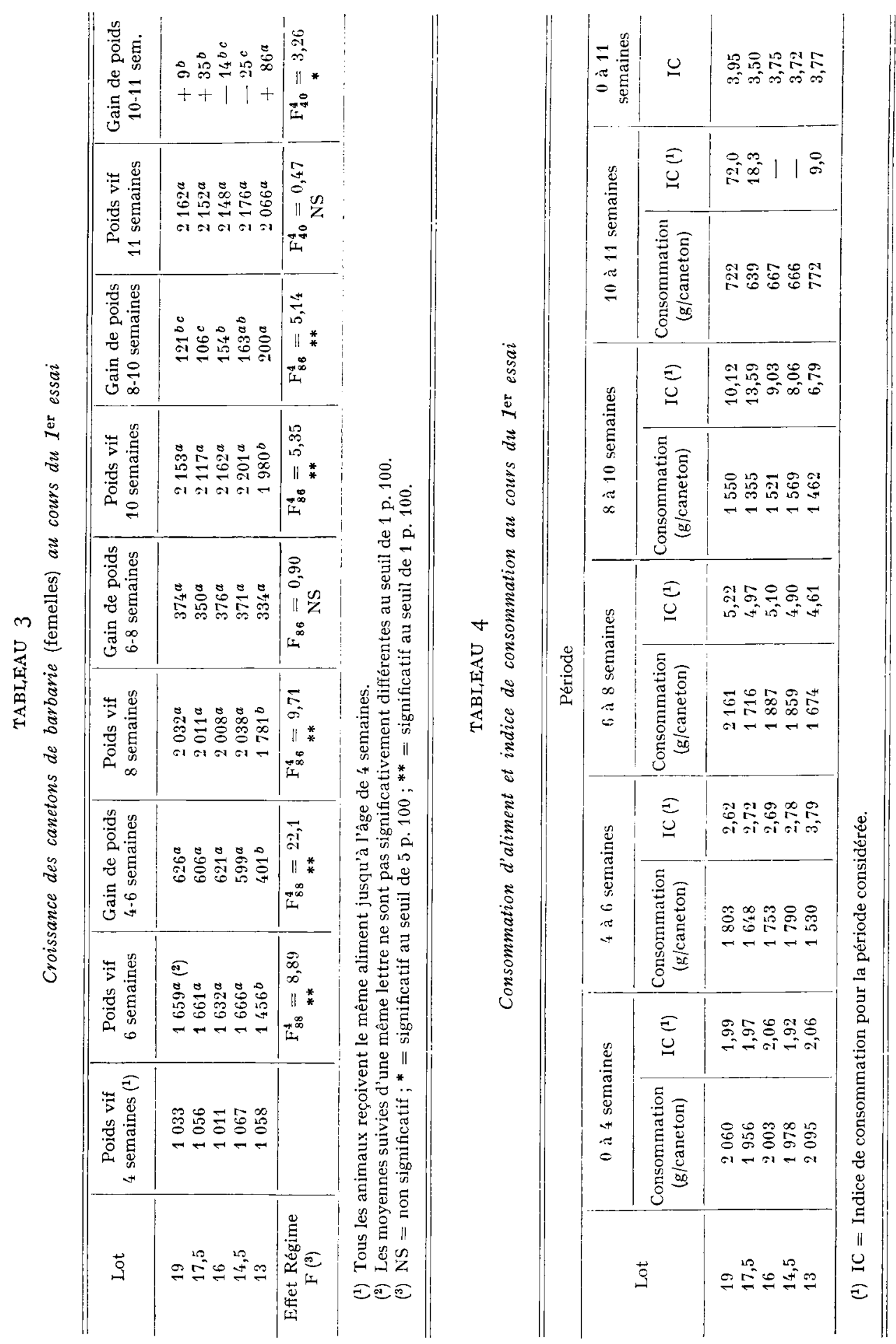


\section{2. - Expérience no 2}

Les résultats de croissance obtenus lors de la $2^{\mathrm{e}}$ expérience sont rapportés dans le tableau 6 . Les animaux du lot I2 (le plus pauvre en MAT) accusent à 6 semaines un retard de croissance de l'ordre de $200 \mathrm{~g}$. Ce retard persiste jusqu'à l'âge de 9 semaines et disparaît ensuite. Toutefois si l'on observe les gains de poids vif réalisés au cours de chaque période on n'enregistre plus de différences entre les lots de 6 à 8 semaines, ni de 8 à 9 semaines. Le lot recevant le moins de protéines présente même entre 9 et ro semaines le gain de poids le plus élevé.

Dans le tableau 7 nous rassemblons les consommations d'aliment et les indices de consommation des divers lots à tous les stades de l'expérience. Seul le régime le plus pauvre en MAT induit une sous-consommation qui s'estompe après l'âge de 8 semaines. Entre 4 et 6 semaines les canetons qui ingèrent cet aliment ont un indice de consommation moins bon que ceux des autres lots. Par la suite ce phénomène disparaît et comme précédemment on enregistre une amélioration de l'indice pendant la phase de croissance compensatrice. L'indice global n'est guère différent d'un lot à l'autre.

Le tableau 8 contient les résultats de dissection réalisés aux âges de 9 et ro semaines. Comme dans le $\mathrm{I}^{\mathrm{r}}$ essai, le rendement à $1^{\prime}$ abattage n'est pas influencé significativement par la nature du régime, ni par l'âge d'abattage. Le poids des cuisses, lui aussi, est indépendant du régime et du stade d'abattage. Seul le poids des filets est influencé par des deux facteurs. Il est plus faible à 9 semaines chez les canards du lot disposant du régime le plus pauvre en MAT. A Io semaines cette différence n'est plus significative. On remarque par ailleurs qu'entre 9 et ro semaines le poids des filets augmente dans tous les lots de ro grammes en moyenne.

\section{DISCUSSION}

Les résultats acquis au cours de ces deux expériences nous permettent de tirer des conclusions à propos d'une part du besoin protéique de la cane de Barbarie en croissance, d'autre part de l'évolution des caractéristiques anatomiques de la carcasse.

\section{Le besoin protéique du caneton}

Les exigences du caneton de Barbarie diminuent notablement avec 1'âge. C'est ainsi que lors du premier essai on peut estimer que le besoin est entre 13,2 et I4,6 p. Ioo de MAT pour la période de 4 à 6 semaines. Il est ensuite voisin de 13,2 p. Ioo et descend en-dessous de cette valeur après 1'âge de 8 semaines. Ces estimations sont valables pour des régimes à base de maïs et soja renfermant I2,5 $\mathrm{MJ}$ d'énergie métabolisable par $\mathrm{kg}$.

I,es résultats du I ${ }^{\text {er }}$ essai sont confirmés par le second. Cette fois-ci le besoin se situe entre I4,6 et I5,5 p. Ioo de MAT pour la période de 4 à 6 semaines; il en est de même entre 6 et 8 semaines. Au-delà le besoin est certainement en-dessous de I2,8 p. roo. Les valeurs du besoin déterminées lors de ce second essai sont un peu supérieures à celles dı premier. La température des locaux peut expliquer cette diffé- 


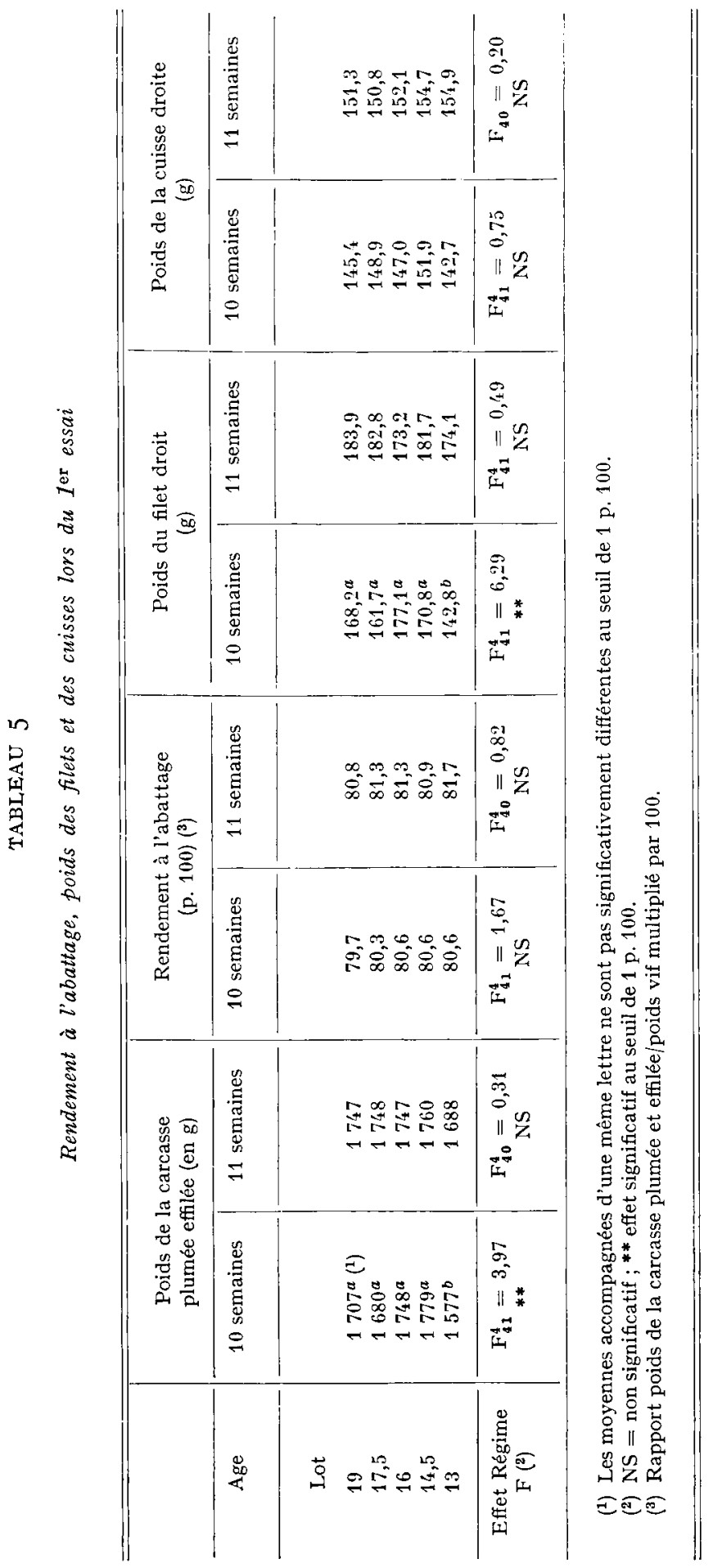


B. LECLERCQ, H. de CARVILLEE

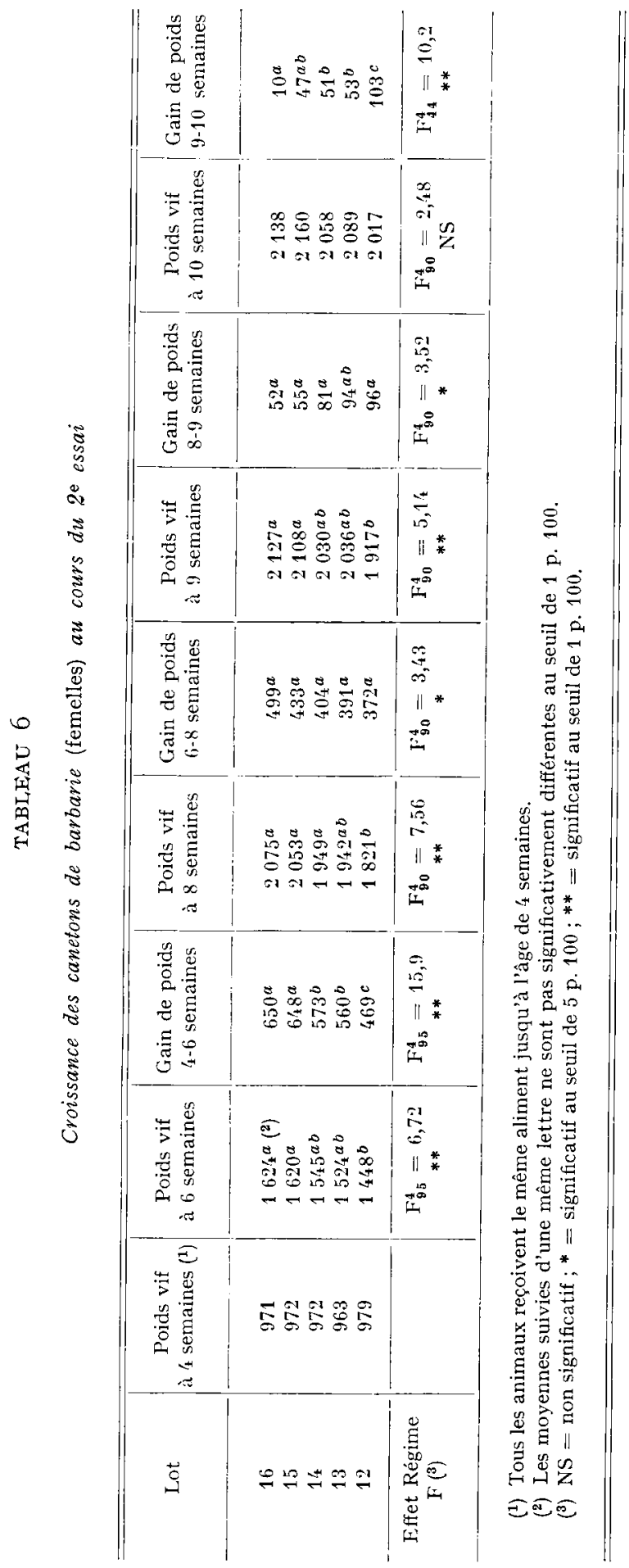



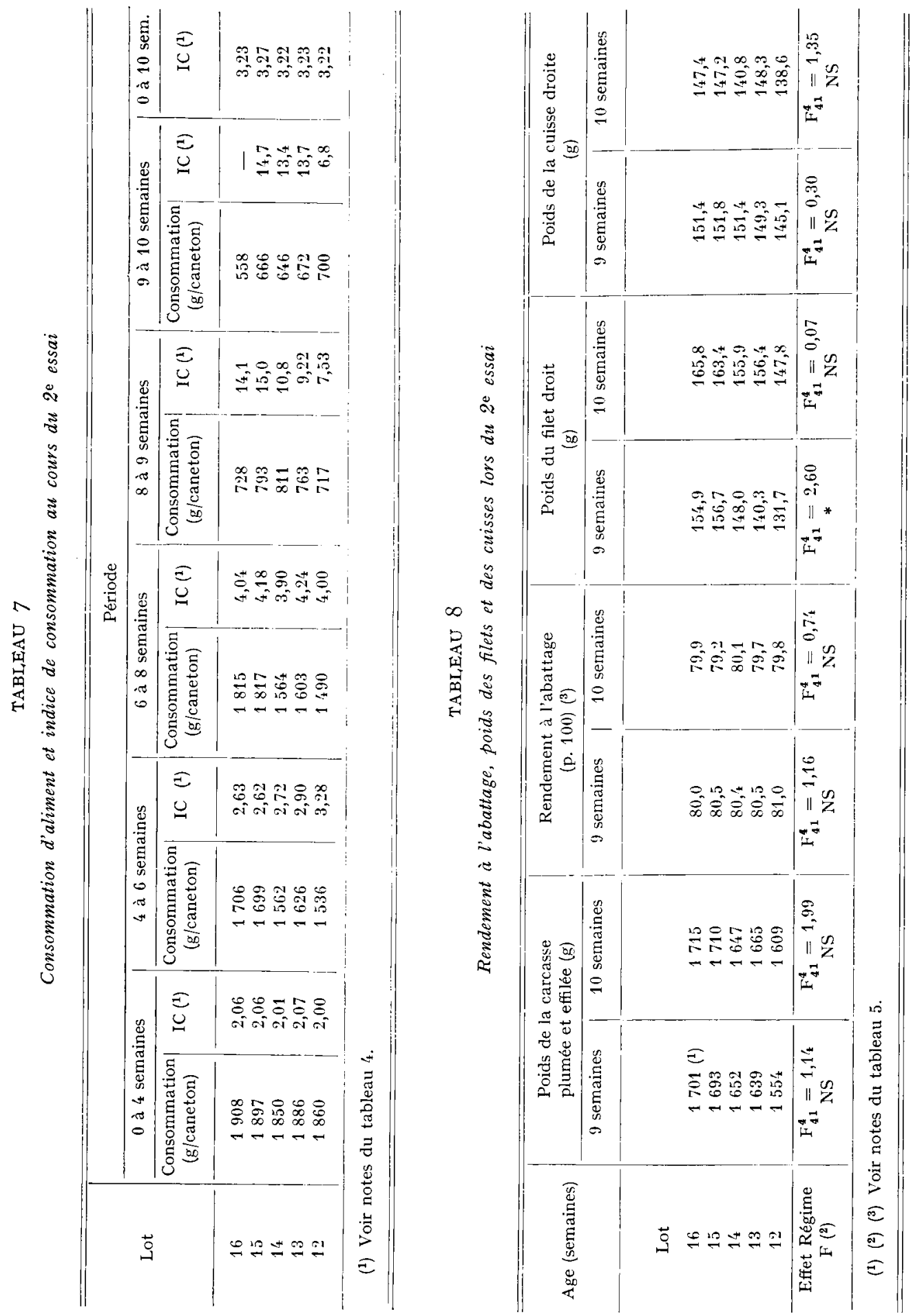
rence. En effet la première expérience a été réalisée au cours d'un printemps froid, alors que la seconde s'est déroulée l'été ; on constate grâce aux tableaux 4 et 7 qu'en saison chaude le caneton consomme moins d'aliment et présente un meilleur indice de consommation. Il lui est cependant indispensable de trouver dans son régime les protéines nécessaires à sa croissance. C'est pourquoi en ambiance chaude la teneur du régime en MAT doit être supérieure à celle de l'ambiance froide.

Nos résultats nous permettent de formuler quelques remarques à propos du comportement alimentaire du canard. En effet contrairement au Poulet le Canard ne réagit pas à l'abaissement de la concentration en protéines du régime par une surconsommation alimentaire. On ne constate pas non plus d'amélioration de l'indice de consommation à mesure que la teneur en MAT s'élève.

\title{
Caractéristiques anatomiques de la carcasse (tabl. 5 et 8)
}

La carence protéique retentit sur le développement des différentes parties de la carcasse. La cuisse n'est jamais affectée dans nos essais. Au contraire le filet peut être moins développé en cas de carence. Toutefois avec l'âge le caneton parvient à compenser ce retard. On doit reconnaître là un simple effet de la carence protéique sur le développement corporel. Chez les palmipèdes en effet les membres postérieurs se développent plus précocement que les membres antérieurs (LUHMANN, I967).

Les masses musculaires des filets ne progressent pas seulement en valeur absolue mais aussi en valeur relative. Enn effet à Io semaines d'âge elles représentent dans le premier essai I $9,7 \mathrm{p}$. Ioo du poids de la carcasse effilée pour les 4 lots recevant les régimes les plus riches en protéines. A l'âge de II semaines cette proportion s'élève à 20,6 p. I0o, l'augmentation étant systématiquement observée dans tous les lots. De même dans le second essai la proportion des filets est de 17,9 p. Ioo à l'âge de 9 semaines. Elle s'accroît dans tous les lots et devient en moyenne de I 9,3 p. Ioo à Io semaines.

Ces observations doivent être prises en considération pour fixer l'âge d'abattage des animaux. En effet les tableaux 4 et 7 révèlent que l'indice de consommation se dégrade très vite chez le Canard ; en particulier vers l'âge de 8 semaines il atteint des valeurs d'indice partiel très supérieures à Io. En raisonnant sur le seul poids vif de l'animal on est logiquement tenté d'avancer l'âge du sacrifice afin d'éviter le gaspillage d'aliment occasionné par les valeurs excessives de l'indice partiel de consommation. Cependant le rendement de la carcasse en parties comestibles s'accroît durant cette période. Elles représentent la majeure partie du gain de poids. L'âge d'abattage dépend donc de l'importance que l'on attribue aux critères quantitatifs et aux critères qualitatifs.

$$
\text { Rę̧u pour publication en janvier } 1975 .
$$

\section{SUMMARY}

\author{
PROTEIN REQUIREMENT OF THE MUSCOVY DUCKLING BETWEEN 4
}

AND II WEEKS OF AGE

Two experiments were made with female Muscovy ducklings (Cairina moschata) between 4 and I I weeks of age. During the first experiment (basal diets given in table $I$, the influence of 5 different crude protein levels was studied : 18.9, 17.4, 16.0, 1 4.6 and I 3.2 p. Ioo. The ducklings 
were sacrified at the age of ro and i i weeks. In the second experiment, 5 other crude protein levels were examined : $16.4,15.5,14.6,13.7$ and $12.8 \mathrm{p}$. I 00 (diets given in table 2 ).

Results concerning growth are shown in tables 3 and 6 , those concerning feed intake are reported in tables 4 and 7 . According to these data, the protein requirement of the duckling represents I 5 p. Ioo between 4 and 6 weeks, I 4.5 p. Ioo between 6 and 8 weeks and is lower than r 3 p. Ioo after that age.

Contrarily to chicken, ducklings do not reduce their feed intake when the dietary protein level increases; consequently, the feed conversion ratio does not depend on the protein level.

The dissection results showed that the maximum weight of the legs was already reached at the age of 9 weeks. Conversely, the absolute and relative value of the breast muscles increased with the age of the animal (tables 5 and 8 ).

\section{RÉFÉRENCES BIBLIOGRAPHIQUES}

Delpech P., Milovanovic M., 1973. Appréciation de la composition corporelle du Canard. Journées Rech. Avic. Cunic., Paris, 29I-295, I. T. A. V. I. éd.

Lïhmans M., I967. Rassenkreuzungen bei Ënten sur Produktion leichter Mastenten. Arch. für Geflügelk., 31, 318-34I. 